PROCEEDINGS OF THE

AMERICAN MATHEMATICAL SOCIETY

Volume 134, Number 8, Pages 2169-2178

S 0002-9939(06)08094-4

Article electronically published on March 20, 2006

\title{
RANK-ONE COMPLETIONS OF PARTIAL MATRICES AND COMPLETELY RANK-NONINCREASING LINEAR FUNCTIONALS
}

\author{
DON HADWIN, K. J. HARRISON, AND J. A. WARD
}

(Communicated by David R. Larson)

\begin{abstract}
We obtain necessary and sufficient conditions for the existence and the uniqueness of rank-one completions of a partial matrix, and we verify a conjecture of Hadwin and Larson concerning the nature of completely ranknonincreasing linear functionals defined on pattern subspaces.
\end{abstract}

\section{Matrix COMPLEtion PROBlems}

Let $\mathcal{P}$ be a pattern, that is, a subset of $\widetilde{m} \times \widetilde{n}=\{1,2, \ldots, m\} \times\{1,2, \ldots, n\}$, where $m$ and $n$ are positive integers. A $\mathcal{P}$-matrix, or partial matrix supported on $\mathcal{P}$, is a complex-valued function $A$ with domain $\mathcal{P}$. We write $A=\left(a_{i j}\right)$ where each entry $a_{i j}$ is the image of $(i, j)$ under $A$, and is defined only for $(i, j) \in \mathcal{P}$. We display partial matrices as ordinary matrices but with missing entries. For example, $A=\left(\begin{array}{ll}1 & 1 \\ & 1\end{array}\right)$ is a partial matrix supported on the pattern $\{(1,1),(1,2),(2,2)\}$, which we display as $\left(\begin{array}{cc}* & * \\ & *\end{array}\right)$.

It is useful to regard $\mathcal{P}$ as the edge set of a bipartite graph that has $m$ row vertices and $n$ column vertices. We shall use the same symbol $\mathcal{P}$ to denote this graph. It should be clear from the context whether we are referring to the pattern or the graph. The entries $a_{i j}$ of any $\mathcal{P}$-matrix $A$ can be regarded as weights associated with the edges in the graph $\mathcal{P}$.

Suppose that $A=\left(a_{i j}\right)$ and $B=\left(b_{i j}\right)$ are $m \times n$ partial matrices with supports $\mathcal{P}$ and $\mathcal{Q}$ respectively. We write $A \preceq B$ and say that $B$ is an extension of $A$, or that $A$ is a restriction of $B$, if $\mathcal{P} \subseteq \mathcal{Q}$ and $a_{i j}=b_{i j}$ for all $(i, j) \in \mathcal{P}$. We say that $B$ is a completion of $A$ if $A \preceq B$ and if $\mathcal{Q}=\widetilde{m} \times \widetilde{n}$, that is, if $B$ is a 'full' $m \times n$ matrix that agrees with $A$ on $\mathcal{P}$. Matrix completion problems are concerned with finding completions of partial matrices that have some prescribed properties. For example, we might want to find completions that are positive definite, or have a given set of eigenvalues, or we might want to find, amongst all possible completions of a given partial matrix, ones with minimal rank or minimal operator norm. Problems of this type have attracted a good deal of attention over the past twenty or so years, and there is an extensive literature devoted to the subject, which can be accessed

Received by the editors April 23, 2002 and, in revised form, July 15, 2004.

2000 Mathematics Subject Classification. Primary 15A60.

(C)2006 American Mathematical Society 
readily via a web search for 'matrix completions'. The survey papers [1, [2], 3], [4], and [5], would give the reader an indication of some of the main results in the field. However the list is by no means exhaustive.

\section{RANK-ONE COMPLETIONS}

In the first part of this paper we study rank completions and obtain necessary and sufficient conditions for the existence and for the uniqueness of a rank-one completion of a given partial matrix. Theorem [5, the key result of this section, is not new and appeared first in [6]. However, our proof is somewhat more transparent and provides a useful setting for our limit results in Section 3 and our application to completely rank-nonincreasing functionals in Section 4 .

The first of our two necessary conditions for the existence of a rank-one completion of a partial matrix $A=\left(a_{i j}\right)$ concerns the location of zeros of $A$, and the second is a relation on the values $a_{i j}$ on any cycle in the graph $\mathcal{P}$.

2.1. The zero row or column property. Any $m \times n$ rank-one matrix can be expressed as a product $e f^{T}$, where $e=\left(e_{i}\right)$ and $f=\left(f_{j}\right)$ are non-zero column vectors in $\mathbf{C}^{m}$ and $\mathbf{C}^{n}$ respectively, and where $f^{T}$ denotes the transpose of $f$.

Suppose that $e f^{T}$ is a rank-one completion of $A$. Then $a_{i j}=e_{i} f_{j}$ for each $(i, j) \in \mathcal{P}$. So if $a_{i j}=0$ for some $(i, j) \in \mathcal{P}$, then $e_{i}=0$ or $f_{j}=0$, and so either $a_{i j^{\prime}}=0$ for all $\left(i, j^{\prime}\right) \in \mathcal{P}$ or $a_{i^{\prime} j}=0$ for all $\left(i^{\prime}, j\right) \in \mathcal{P}$. In other words, if any entry of $A$ is zero, then either every other entry of $A$ in the same row is zero or every other entry in the same column is zero. We say that a partial matrix $A=\left(a_{i j}\right)$ has the zero row or column property if

$$
a_{i j}=0 \Rightarrow\left(a_{i j^{\prime}}=0 \text { for all }\left(i, j^{\prime}\right) \in \mathcal{P} \text { or } a_{i^{\prime} j}=0 \text { for all }\left(i^{\prime}, j\right) \in \mathcal{P}\right) .
$$

Thus we have established the following simple necessary condition for the existence of a rank-one completion of a partial matrix.

Lemma 1. If a partial matrix has a rank-one completion, then it has the zero row or column property.

2.2. The cycle property. Let $\Gamma$ be a directed path in $\mathcal{P}$ starting at vertex $\alpha$ and ending at vertex $\omega$. Thus $\Gamma$ consists of a sequence of vertices $v(0), v(1), v(2), \ldots$, $v(K)$ with $v(0)=\alpha$ and $v(K)=\omega$ that are adjacent in $P$, that is, for $1 \leq i<K$,

$$
\begin{aligned}
& (v(i), v(i+1)) \in \mathcal{P} \text { if } v(i) \text { is a row vertex, and } \\
& (v(i+1), v(i)) \in \mathcal{P} \text { if } v(i) \text { is a column vertex. }
\end{aligned}
$$

We shall associate with the path $\Gamma$ and the partial matrix $A$ two numbers $\Gamma_{R C}(A)$ and $\Gamma_{C R}(A)$, which we call the alternating edges products of $A$ along $\Gamma$ :

$$
\Gamma_{R C}(A)=\prod_{v(i) \in \widetilde{m}} a_{v(i), v(i+1)} \quad \text { and } \quad \Gamma_{C R}(A)=\prod_{v(i) \in \widetilde{n}} a_{v(i+1), v(i)} .
$$

Thus $\Gamma_{R C}(A)$ is the product of the edge weights taken over all edges $(v(i), v(i+1))$ in $\Gamma$ for which $v(i)$ is a row vertex, and $\Gamma_{C R}(A)$ is the product of the edge weights taken over all edges $(v(i), v(i+1))$ in $\Gamma$ for which $v(i)$ is a column vertex. We set $\Gamma_{R C}(A)=1$ if there is no edge in $\Gamma$ from a row vertex to a column vertex and similarly for $\Gamma_{C R}(A)$. 
If $-\Gamma$ denotes the same path as $\Gamma$, but traversed in the reverse order, then row-to-column edges become column-to-row edges and vice versa. So we have

$$
(-\Gamma)_{R C}(A)=\Gamma_{C R}(A) \text { and }(-\Gamma)_{C R}(A)=\Gamma_{R C}(A) .
$$

Furthermore, if $\Gamma$ is the concatenation of two paths $\Gamma^{\prime}$ and $\Gamma^{\prime \prime}$, where the end vertex of $\Gamma^{\prime}$ is the start vertex of $\Gamma^{\prime \prime}$, then it is easy to verify that

$$
\Gamma_{R C}(A)=\Gamma_{R C}^{\prime}(A) \Gamma_{R C}^{\prime \prime}(A) \text { and } \Gamma_{C R}(A)=\Gamma_{C R}^{\prime}(A) \Gamma_{C R}^{\prime \prime}(A) .
$$

Suppose now that $\Gamma$ is a cycle, that is, a path starting and ending at the same vertex $\alpha$, which, for convenience, we shall assume is a row vertex. Also since the number of edges in $\Gamma$ is even, $K=2 k$ for some positive integer $k$, and $v(i)$ is a row vertex if $i$ is even and $v(i)$ is a column vertex if $i$ is odd. Then $\Gamma_{R C}(A)=$ $\prod_{i=0}^{k-1} a_{v(2 i), v(2 i+1)}$ and $\Gamma_{C R}(A)=\prod_{i=1}^{k} a_{v(2 i), v(2 i-1)}$. Suppose also that $e f^{T}$ is a rank-one completion of $A$. Since $a_{i j}=e_{i} f_{j}$ for each $(i, j) \in \mathcal{P}$, it follows that

$$
\Gamma_{R C}(A)=\prod_{i=0}^{k-1} e_{v(2 i)} f_{v(2 i+1)} \text { and } \Gamma_{C R}(A)=\prod_{i=1}^{k} e_{v(2 i)} f_{v(2 i-1)} \text {. }
$$

Since $\alpha=v(0)=v(2 k)$, the alternating edge products are equal, that is,

$$
\Gamma_{R C}(A)=\Gamma_{C R}(A) .
$$

We call (5) the cycle property, and we say that $A$ has the cycle property if it satisfies (5) on all cycles $\Gamma$ in its support $\mathcal{P}$. Thus we have established the following result.

Lemma 2. If a partial matrix has a rank-one completion, then it has the cycle property.

The following result will also be useful.

Lemma 3. Suppose that the partial matrix $A$ has the cycle property and that $\Gamma$ and $\Gamma^{\prime}$ are paths in $\mathcal{P}$ with the same start vertex and the same end vertex. Then

$$
\Gamma_{R C}(A) \Gamma_{C R}^{\prime}(A)=\Gamma_{C R}(A) \Gamma_{R C}^{\prime}(A) .
$$

Proof. Let $\Gamma^{\#}$ denote the concatenation of $\Gamma$ and $-\Gamma^{\prime}$. Then $\Gamma^{\#}$ is a closed path, and so by the cycle property $\Gamma_{R C}^{\#}(A)=\Gamma_{C R}^{\#}(A)$. But

$$
\Gamma_{R C}^{\#}(A)=\Gamma_{R C}(A)\left(-\Gamma^{\prime}\right)_{R C}(A)=\Gamma_{R C}(A) \Gamma_{C R}^{\prime}(A)
$$

and

$$
\Gamma_{C R}^{\#}(A)=\Gamma_{C R}(A)\left(-\Gamma^{\prime}\right)_{C R}(A)=\Gamma_{C R}(A) \Gamma_{R C}^{\prime}(A)
$$

by (3) and (4).

2.3. Components. We shall show that the zero row or column property, together with the cycle property, are sufficient for the existence of a rank-one completion, but first we show that it is sufficient to consider partial matrices defined on connected graphs.

We say that the partial matrix $A$ is a component of the partial matrix $B$ if $A \preceq B$ and if $\mathcal{P}$ is a connected component of the graph $\mathcal{Q}$. If $A$ is a component of $B$, then after a possible reordering of the rows and columns, $B$ may be written in block matrix form

$$
B=\left(\begin{array}{ll}
A & \\
& C
\end{array}\right),
$$


where $C$ is a partial matrix supported on $\mathcal{Q} \backslash \mathcal{P}$. Suppose that $B$ has a rank-one completion $R=e f^{T}$, where $e$ and $f$ have block matrix form $e=\left(\begin{array}{c}\varepsilon_{1} \\ \varepsilon_{2}\end{array}\right)$ and $f=\left(\begin{array}{l}\varphi_{1} \\ \varphi_{2}\end{array}\right)$. Then

$$
R=e f^{T}=\left(\begin{array}{ll}
\varepsilon_{1} \varphi_{1}^{T} & \varepsilon_{1} \varphi_{2}^{T} \\
\varepsilon_{2} \varphi_{1}^{T} & \varepsilon_{2} \varphi_{2}^{T}
\end{array}\right),
$$

and it is clear that $\varepsilon_{1} \varphi_{1}^{T}$ and $\varepsilon_{2} \varphi_{2}^{T}$ are completions of $A$ and $C$ respectively with ranks at most 1. Furthermore if $A$ is non-zero, then the rank of $\varepsilon_{1} \varphi_{1}^{T}$ is 1 .

Conversely, if $\varepsilon_{1} \varphi_{1}^{T}$ and $\varepsilon_{2} \varphi_{2}^{T}$ are rank-one completions of $A$ and $C$ respectively, then the rank-one matrix

$$
R_{\lambda}=\left(\begin{array}{c}
\varepsilon_{1} \\
\lambda \varepsilon_{2}
\end{array}\right)\left(\begin{array}{c}
\varphi_{1} \\
\lambda^{-1} \varphi_{2}
\end{array}\right)^{T}=\left(\begin{array}{cc}
\varepsilon_{1} \varphi_{1}^{T} & \lambda^{-1} \varepsilon_{1} \varphi_{2}^{T} \\
\lambda \varepsilon_{2} \varphi_{1}^{T} & \varepsilon_{2} \varphi_{2}^{T}
\end{array}\right)
$$

is a completion of $B$ for any non-zero complex number $\lambda$. These ideas can be easily extended to prove the following.

Lemma 4. A non-zero partial matrix has a rank-one completion if and only if each of its non-zero components has a rank-one completion.

We now prove the main theorem of this section.

Theorem 5. A non-zero partial matrix has a rank-one completion if and only if it has the zero row or column property (10) and the cycle property (5).

Proof. Suppose that $A=\left(a_{i j}\right)$ is a $\mathcal{P}$-matrix that has the zero row or column property and the cycle property. By Lemmas (11) and (2) it is sufficient to prove that $A$ has a rank-one completion. After a possible reordering of the rows and columns we may assume that $A$ has the block matrix form

$$
A=\left(\begin{array}{cc}
0 & 0 \\
0 & A^{\#}
\end{array}\right)
$$

where $A^{\#}$ is a restriction of $A$ whose support is $\mathcal{P}^{\#}=\left\{(i, j) \in \mathcal{P}: a_{i j} \neq 0\right\}$, and where each 0 denotes a partial matrix with no non-zero entry (and possibly no entry at all). Clearly if $R^{\#}$ is a rank-one completion of $A^{\#}$, then $\left(\begin{array}{cc}0 & 0 \\ 0 & R^{\#}\end{array}\right)$ is a rank-one completion of $A$. (Here 0 denotes a 'full' matrix whose entries are all 0 .) So we may assume from the outset that $a_{i j} \neq 0$ for all $(i, j) \in \mathcal{P}$, that is, $\mathcal{P}=\mathcal{P}^{\#}$. Furthermore, by Lemma 4, we may assume that $\mathcal{P}$ is connected.

We shall construct vectors $e=\left(e_{i}\right) \in \mathbf{C}^{m}$ and $f=\left(f_{j}\right) \in \mathbf{C}^{n}$ such that $a_{i j}=e_{i} f_{j}$ for each $(i, j) \in \mathcal{P}$. For each vertex $v \in \mathcal{P}$ let $\Gamma$ be a path in $\mathcal{P}$ starting at row vertex 1 and ending at $v$, and define

$$
\left\{\begin{array}{l}
e_{v}=\Gamma_{C R}(A) / \Gamma_{R C}(A) \quad \text { if } v \text { is a row vertex } \\
f_{v}=\Gamma_{R C}(A) / \Gamma_{C R}(A) \quad \text { if } v \text { is a column vertex. }
\end{array}\right.
$$

We need to show that the vectors $e$ and $f$, whose components $e_{v}$ and $f_{v}$ are defined as in (6), have the desired properties.

To see that (6) provides a formula for defining each $e_{i}$ and $f_{j}$ uniquely, first observe that the connectedness of $\mathcal{P}$ guarantees that there is a path from row 
vertex 1 to any other vertex $v$. Next suppose that $\Gamma$ and $\Gamma^{\prime}$ are two paths starting at row vertex 1 and ending at a vertex $v$. Then by Lemma 3 ,

$$
\Gamma_{C R}(A) / \Gamma_{R C}(A)=\Gamma_{C R}^{\prime}(A) / \Gamma_{R C}^{\prime}(A) .
$$

So the ratio $\Gamma_{C R}(A) / \Gamma_{R C}(A)$ is independent of the choice of the path from 1 to $v$, and hence $e_{v}$ and $f_{v}$ are indeed well-defined.

Finally suppose that $(i, j) \in \mathcal{P}$, let $\Gamma$ be a path in $\mathcal{P}$ starting at row vertex 1 and ending at row vertex $i$, and let $\Gamma^{\prime}$ be the concatenation of $\Gamma$ with the edge $(i, j)$. Then

$$
\Gamma_{R C}^{\prime}(A)=\Gamma_{R C}(A) \times a_{i j} \text { and } \Gamma_{C R}^{\prime}(A)=\Gamma_{C R}(A),
$$

and so

$$
a_{i j}=\frac{\Gamma_{R C}^{\prime}(A)}{\Gamma_{R C}(A)}=\frac{\Gamma_{C R}(A)}{\Gamma_{R C}(A)} \times \frac{\Gamma_{R C}^{\prime}(A)}{\Gamma_{C R}^{\prime}(A)}=e_{i} f_{j} .
$$

So the rank-one matrix $e f^{T}$ is indeed a completion of $A$.

Corollary 6. A non-zero $\mathcal{P}$-matrix $A$ has a unique rank-one completion if and only if it has the zero row or column property (1) and the cycle property (15), and $\mathcal{P}^{\#}=\left((i, j): a_{i j} \neq 0\right\}$ is connected.

Proof. Suppose that $A$ has the zero row or column property and the cycle property. Then by Theorem $5 A$ has a rank-one completion, and so we need to show that this completion is unique if and only if $\mathcal{P}^{\#}$ is connected.

If $\mathcal{P}^{\#}$ is not connected, then the remarks in subsection 2.3 show that $A$ has infinitely many rank-one completions. So suppose that $\mathcal{P}^{\#}$ is connected, and let $e$ and $f$ denote the vectors whose components $e_{v}$ and $f_{v}$ are defined as in (6). Then, as in the proof of Theorem 5 , $e f^{T}$ is a rank-one completion of $A$. To see that $e f^{T}$ is unique, choose any $(i, j) \in \widetilde{m} \times \widetilde{n}$. If $(i, j) \in \mathcal{P}$, then $e_{i} f_{j}=a_{i j}$ by (7). If $(i, j) \notin \mathcal{P}$, let $\Gamma$ be a path in $\mathcal{P}$ starting at row vertex $i$ and ending at column vertex $j$, and let $\Gamma^{\prime}$ consist of the single edge $(i, j)$. Since $e f^{T}$ has rank one, it has the cycle property of the complete graph $\widetilde{m} \times \widetilde{n}$, and so by Lemma 3 ,

$$
\Gamma_{R C}\left(e f^{T}\right) \Gamma_{C R}^{\prime}\left(e f^{T}\right)=\Gamma_{R C}^{\prime}\left(e f^{T}\right) \Gamma_{C R}\left(e f^{T}\right) .
$$

Now $\Gamma_{R C}\left(e f^{T}\right)=\Gamma_{R C}(A)$ and $\Gamma_{C R}\left(e f^{T}\right)=\Gamma_{C R}(A)$ since $e f^{T}$ is a completion of $A$, and $\Gamma_{C R}^{\prime}\left(e f^{T}\right)=1$ and $\Gamma_{R C}^{\prime}\left(e f^{T}\right)=e_{i} f_{j}$. So

$$
e_{i} f_{j}=\Gamma_{R C}(A) / \Gamma_{C R}(A)
$$

that is, each product $e_{i} f_{j}$ is uniquely defined as a ratio of products of non-zero entries $a_{i j}$ of the partial matrix $A$. Hence the rank-one completion of $A$ is unique.

\section{LIMITS OF RANK-ONE MATRICES}

The set of all $\mathcal{P}$-matrices for a given pattern $\mathcal{P}$ is a normed vector space under 'pointwise' addition and scalar multiplication and with norm

$$
\|A\|_{\mathcal{P}}=\sup _{(i, j) \in \mathcal{P}}\left|a_{i j}\right| \text {. }
$$

We write $A^{(k)} \rightarrow_{\mathcal{P}} A$ as $k \rightarrow \infty$ if $A$ and each $A^{(k)}$ is a $\mathcal{P}$-matrix, and if $\left\|A-A^{(k)}\right\|_{\mathcal{P}}$ $\rightarrow 0$ as $k \rightarrow \infty$. Let $\mathfrak{F}_{1}(\mathcal{P})$ denote the set of all $\mathcal{P}$-matrices that are restrictions of matrices of the form $e \otimes f$, that is, the set of all $\mathcal{P}$-matrices that have completions 
with rank at most 1 . If $\mathcal{P}=\widetilde{m} \times \widetilde{n}$, then $\mathfrak{F}_{1}(\mathcal{P})$ is closed, but this is not the case for all patterns $\mathcal{P}$ as the following example demonstrates.

Example 7. Let $A$ be the partial matrix $\left(\begin{array}{ll}1 & 0 \\ & 1\end{array}\right)$, where $\mathcal{P}=\left(\begin{array}{cc}* & * \\ & *\end{array}\right)$. Then $A \notin \mathfrak{F}_{1}(\mathcal{P})$ because it does not have the zero row or column property (11). But $A-\left(\begin{array}{c}1 \\ 1 / k \\ 1\end{array}\right) \rightarrow_{\mathcal{P}} A$ as $k \rightarrow \infty$, and $\left(\begin{array}{cc}1 & 1 / k \\ 1\end{array}\right)$ has a (unique) rank-one completion $\left(\begin{array}{cc}1 & 1 / k \\ k & 1\end{array}\right)$ for each $k \geq 1$.

We say that a partial matrix in $\overline{\mathfrak{F}_{1}(\mathcal{P})}$, the closure of $\mathfrak{F}_{1}(\mathcal{P})$, is a partial limit of rank-one matrices. Example 7 shows that partial limits of rank-one matrices do not necessarily have the zero row or column property (10). On the other hand the following simple argument shows that they do have the cycle property (5). Suppose that $A \in \overline{\mathfrak{F}_{1}(\mathcal{P})}$. Then there is a sequence of rank-one matrices $R^{(k)}, k=1,2,3, \ldots$, such that $R^{(k)} \rightarrow \mathcal{P} A$ as $k \rightarrow \infty$. Now let $\Gamma$ be a cycle in $\mathcal{P}$. Since each $R^{(k)}$ has the cycle property, $\Gamma_{R C}\left(R^{(k)}\right)=\Gamma_{C R}\left(R^{(k)}\right)$ for each $k$. But $\Gamma_{R C}\left(R^{(k)}\right) \rightarrow \Gamma_{R C}(A)$ and $\Gamma_{C R}\left(R^{(k)}\right) \rightarrow \Gamma_{C R}(A)$ as $k \rightarrow \infty$, and so $\Gamma_{R C}(A)=\Gamma_{C R}(A)$. Thus $A$ has the cycle property.

The main result of this section shows that the cycle property characterizes the set of partial limits of rank-one matrices.

Theorem 8. A partial matrix is a partial limit of rank-one matrices if and only if it has the cycle property (5).

Proof. Suppose that $A$ is a $\mathcal{P}$-matrix that has the cycle property. If $A=0$, let $R$ be an arbitrary rank-one matrix and let $R_{\mathcal{P}}$ denote its restriction to $\mathcal{P}$. Then $\frac{1}{k} R_{\mathcal{P}} \rightarrow \mathcal{P} 0$ as $k \rightarrow \infty$.

So we may assume that $A$ has some non-zero entries. After possibly reordering the row and column vertices, we may suppose that $A$ has the block matrix form

$$
A=\left(\begin{array}{cc}
0 & 0 \\
0 & A^{\prime}
\end{array}\right)
$$

where $A^{\prime}$ is a restriction of $A$ with the property that each row and column of $A^{\prime}$ contains a non-zero entry, and where each 0 denotes a zero partial matrix. Clearly if $R^{\prime}$ is a rank-one completion of $A^{\prime}$, then $\left(\begin{array}{cc}0 & 0 \\ 0 & R^{\prime}\end{array}\right)$ is a rank-one completion of $A$. (Here 0 denotes a 'full' matrix whose entries are all 0.) So we may assume from the outset that each row and column contains a non-zero entry of $A$. Furthermore, the proof of Lemma 4 can easily be modified to show that a partial matrix is a partial limit of rank-one matrices if and only if each of its components is a partial limit of rank ones. So we may assume that $\mathcal{P}$ is connected.

Let $\mathcal{P}_{1}, \mathcal{P}_{2}, \ldots, \mathcal{P}_{L}$ denote the connected components of $\mathcal{P}^{\#}=\{(i, j) \in \mathcal{P}$ : $\left.a_{i j} \neq 0\right\}$, and let $A_{1}, A_{2}, \ldots, A_{L}$ denote the corresponding components of $A^{\#}$, the restriction of $A$ to $\mathcal{P}^{\#}$. After reordering the rows and columns if necessary, we may 
assume that $A^{\#}$ has block diagonal form

$$
A^{\#}=\left(\begin{array}{cccc}
A_{1} & & & \\
& A_{2} & & \\
& & \ddots & \\
& & & A_{L}
\end{array}\right) .
$$

We now use the zeros of $A$ to define a relation on the components $\mathcal{P}_{1}, \mathcal{P}_{2}, \ldots, \mathcal{P}_{L}$. Write $\mathcal{P}_{r} \sim \mathcal{P}_{s}$ if $a_{i j}=0$ for some $(i, j) \in \mathcal{P}$ where $i \in \mathcal{P}_{r}$ and $j \in \mathcal{P}_{s}$. Suppose that $\mathcal{P}_{r} \sim \mathcal{P}_{r}$. Then $a_{i j}=0$ for some $(i, j) \in \mathcal{P}$ where $i \in \mathcal{P}_{r}$ and $j \in \mathcal{P}_{r}$. Now $\mathcal{P}_{r}$ is a connected component of $\mathcal{P}^{\#}$, and so there is a cycle $\Gamma$ in $\mathcal{P}$ consisting of the edge $(i, j)$ followed by a path in $\mathcal{P}_{r}$ from column vertex $j$ to row vertex $i$. But this violates the cycle property of $A$, since $\Gamma_{R C}(A)=0$ but $\Gamma_{C R}(A)$ is a product of non-zero weights $a_{u v} \neq 0$. So $\sim$ is anti-reflexive, that is, for each $r, \mathcal{P}_{r} \nsim \mathcal{P}_{r}$.

Next we show that $\sim$ is anti-symmetric. Suppose that $\mathcal{P}_{r} \sim \mathcal{P}_{s}$ and $\mathcal{P}_{s} \sim \mathcal{P}_{r}$. Then $a_{i j}=a_{k \ell}=0$ for some $(i, j)$ and $(k, \ell) \in \mathcal{P}$ where $i, \ell \in \mathcal{P}_{r}$ and $j, k \in \mathcal{P}_{s}$. Since $\mathcal{P}_{r}$ and $\mathcal{P}_{s}$ are connected components of $\mathcal{P}^{\#}$, there is a cycle $\Gamma$ in $\mathcal{P}$ consisting of the edge $(i, j)$, followed by a path in $\mathcal{P}_{s}$ from column vertex $j$ to row vertex $k$, followed by the edge $(k, \ell)$, followed by the path in $\mathcal{P}_{r}$ from column vertex $\ell$ to row vertex $i$. But this too violates the cycle property of $A$, since $\Gamma_{R C}(A)=0$ but $\Gamma_{C R}(A) \neq 0$.

Since $\sim$ is anti-reflexive and anti-symmetric, we may assume, after a possible reordering of components $\mathcal{P}_{\ell}$, that $\sim$ is compatible with the natural ordering, that is, $\mathcal{P}_{r} \sim \mathcal{P}_{s} \Rightarrow r<s$. It then follows that $A$ has block upper triangular form

$$
A=\left(\begin{array}{cccc}
A_{1} & 0 & \cdots & 0 \\
& A_{2} & \cdots & 0 \\
& & \ddots & \vdots \\
& & & A_{L}
\end{array}\right)
$$

where each 0 denotes a partial matrix with no non-zero entry (and possibly no entry at all).

It follows from Theorem 5 and Corollary 6 that each $A_{\ell}$ has a unique rank-one completion, $e_{\ell} f_{\ell}^{T}$. For each $k \geq 1$ let $R(k)$ denote the rank-one matrix given by

$$
R^{(k)}=e(k) f(k)^{T} \text { where } e(k)=\left(\begin{array}{c}
k e_{1} \\
k^{2} e_{2} \\
\vdots \\
k^{L} e_{L}
\end{array}\right) \text { and } f(k)=\left(\begin{array}{c}
k^{-1} f_{1} \\
k^{-2} f_{2} \\
\vdots \\
k^{-L} f_{L}
\end{array}\right)
$$

that is,

$$
R^{(k)}=\left(\begin{array}{cccc}
e_{1} f_{1}^{T} & k^{-1} e_{1} f_{2}^{T} & \cdots & k^{1-L} e_{1} f_{L}^{T} \\
k e_{2} f_{1}^{T} & e_{2} f_{2}^{T} & \cdots & k^{2-L} e_{2} f_{L}^{T} \\
\vdots & \vdots & \ddots & \vdots \\
k^{L-1} e_{L} f_{1}^{T} & k^{L-2} e_{L} f_{2}^{T} & \cdots & e_{L} f_{L}^{T}
\end{array}\right)
$$

Then $R_{\mathcal{P}}^{(k)} \rightarrow_{\mathcal{P}} A$ as $k \rightarrow \infty$. So $A$ is a partial limit of rank-one matrices. 


\section{RANK-NONINCREASING FUNCTIONALS}

Suppose that $\Phi$ is a linear functional defined on a linear space $\mathcal{S}$ of complexvalued $m \times n$ matrices. For each $k \geq 1$ let $M_{k}(\mathcal{S})$ denote the space of all $k \times k$ matrices with $\mathcal{S}$-valued entries, that is, $M_{k}(\mathcal{S})=\left\{\left(S_{i j}\right)_{k \times k}: S_{i j} \in \mathcal{S}\right\}$. We define $\Phi_{k}: M_{k}(\mathcal{S}) \rightarrow M_{k}(\mathbf{C})$ by

$$
\Phi_{k}\left(\left(S_{i j}\right)_{k \times k}\right)=\left(\Phi\left(S_{i j}\right)\right)_{k \times k},
$$

and we say that $\Phi$ is completely rank-nonincreasing if

$$
\operatorname{rank}\left(\Phi_{k}\left(\left(S_{i j}\right)_{k \times k}\right)\right) \leq \operatorname{rank}\left(\left(S_{i j}\right)_{k \times k}\right) \text { for all }\left(S_{i j}\right)_{k \times k} \in M_{k}(\mathcal{S})
$$

There is a simple way of producing completely rank-nonincreasing functionals. We say that $\Phi$ is a skew-compression if there are vectors $e \in \mathbf{C}^{n}$ and $f \in \mathbf{C}^{m}$ such that

$$
\Phi(S)=f^{T} S e \text { for each } S \in \mathcal{S} \text {. }
$$

Suppose that (9) holds and that $S=\left(S_{i j}\right)_{k \times k} \in M_{k}(\mathcal{S})$ for some $k \geq 1$. Then

$$
\begin{aligned}
\Phi_{k}(S)= & \left(\Phi\left(S_{i j}\right)\right)_{k \times k}=\left(f^{T} S_{i j} e\right)_{k \times k} \\
= & \left(\begin{array}{cccc}
f & 0 & \cdots & 0 \\
0 & f & \cdots & 0 \\
\vdots & \vdots & \ddots & \vdots \\
0 & 0 & \cdots & f
\end{array}\right) S\left(\begin{array}{cccc}
e & 0 & \cdots & 0 \\
0 & e & \cdots & 0 \\
\vdots & \vdots & \ddots & \vdots \\
0 & 0 & \cdots & e
\end{array}\right),
\end{aligned}
$$

and so $\operatorname{rank} \Phi_{k}(S) \leq \operatorname{rank} S$. Thus $\Phi$ is completely rank-nonincreasing.

Similar results can be obtained by taking limits. We say that $\Phi$ is a limit of skew-compressions if there is a sequence of skew-compressions $\Phi^{(r)}: r=1,2,3, \ldots$, such that

$$
\Phi(S)=\lim _{r \rightarrow \infty} \Phi^{(r)}(S) \text { for each } S \in \mathcal{S} .
$$

It is easy to verify that any limit of skew-compressions is completely rank-nonincreasing.

The role of completely rank-nonincreasing functionals, and of more general completely rank-nonincreasing linear maps, in the study of operator algebras has been recognized by various authors. The paper [7] is a good survey and contains these basic results concerning skew-compressions and their limits. It also contains the following.

Conjecture 9. Every completely rank-nonincreasing linear functional (or, more generally, linear map) is a limit of skew-compressions.

Conjecture 9 has been verified in the case where the domain $\mathcal{S}$ is a $C^{*}$-algebra [7], and where $\mathcal{S}$ is a nest algebra acting on a separable Hilbert space [8]. In this section we verify the conjecture in the case where the domain is a pattern subspace, as defined below.

The pattern subspace $\mathcal{S}_{\mathcal{P}}$ corresponding to a given pattern $\mathcal{P}$ consists of all $m \times n$ matrices that are supported on $\mathcal{P}$, that is,

$$
\mathcal{S}_{\mathcal{P}}=\left\{S=\left(s_{i j}\right)_{m \times n}: s_{i j}=0 \text { for all }(i, j) \notin \mathcal{P}\right\} .
$$

For $(i, j) \in \widetilde{m} \times \widetilde{n}$, let $E_{i j}$ denote the $m \times n$ matrix with 1 in the $(i, j)$-entry and 0 in every other entry. Then $\left\{E_{i j}:(i, j) \in \mathcal{P}\right\}$ is a basis for $\mathcal{S}_{\mathcal{P}}$. 
Suppose that $\Phi$ is a linear functional defined on $\mathcal{S}_{\mathcal{P}}$. By writing $\Phi=\left(\varphi_{i j}\right)$, where $\varphi_{i j}=\Phi\left(E_{i j}\right)$ for each $(i, j) \in \mathcal{P}$, we can regard $\Phi$ as a $\mathcal{P}$-matrix. Suppose that $e f^{T}$ is a completion of $\Phi$. Then for each $S=\left(s_{i j}\right) \in \mathcal{S}_{\mathcal{P}}$,

$$
\Phi(S)=\Phi\left(\sum_{(i, j) \in \mathcal{P}} s_{i j} E_{i j}\right)=\sum_{(i, j) \in \mathcal{P}} s_{i j} \varphi_{i j}=\sum_{(i, j) \in \mathcal{P}} s_{i j} e_{i} f_{j}=f^{T} S e .
$$

So $\Phi$ is a skew-compression. Conversely, if $\Phi(S)=f^{T} S e$ for all $S \in \mathcal{S}_{\mathcal{P}}$, then $e f^{T}$ is a completion of $\Phi$. Furthermore, by taking limits we can easily prove the following.

Lemma 10. The linear functional $\Phi$ is a limit of skew-compressions on $\mathcal{S}_{\mathcal{P}}$ if and only if the partial matrix $\Phi$ is a partial limit of rank-one matrices.

Suppose now that $\Phi$ is a completely rank-nonincreasing linear functional defined on $\mathcal{S}_{\mathcal{P}}$, and suppose that $\Gamma$ is a simple cycle in $\mathcal{P}$, that is, a closed path in $\mathcal{P}$ whose vertices are all distinct except for the common start and end vertex. By reordering the rows and columns if necessary, we may assume that the edge set of $\Gamma$ is

$\{(1,1),(1,2),(2,2),(2,3),(3,3), \ldots,(k-1, k),(k, k),(k, 1)\}$ for some $k>1$.

Let $S$ denote the matrix whose $k \times k$ block matrix form is

$$
S=\left(\begin{array}{cccccc}
E_{11} & -E_{12} & 0 & \cdots & 0 & 0 \\
0 & E_{22} & -E_{23} & \ddots & \ddots & 0 \\
0 & 0 & E_{33} & \ddots & \ddots & \vdots \\
\vdots & \ddots & \ddots & \ddots & \ddots & 0 \\
0 & \ddots & \ddots & \ddots & E_{k-1, k-1} & -E_{k-1, k} \\
-E_{k 1} & 0 & \cdots & 0 & 0 & E_{k k}
\end{array}\right) .
$$

Then

$$
\Phi_{k}(S)=\left(\begin{array}{cccccc}
\varphi_{11} & -\varphi_{12} & 0 & \cdots & 0 & 0 \\
0 & \varphi_{22} & -\varphi_{23} & \ddots & \ddots & 0 \\
0 & 0 & \varphi_{33} & \ddots & \ddots & \vdots \\
\vdots & \ddots & \ddots & \ddots & \ddots & 0 \\
0 & \ddots & \ddots & \ddots & \varphi_{k-1, k-1} & -\varphi_{k-1, k} \\
-\varphi_{k 1} & 0 & \cdots & 0 & 0 & \varphi_{k k}
\end{array}\right) .
$$

It is easy to check that $\operatorname{rank} S=k-1$, and since $\Phi$ is assumed to be completely rank-nonincreasing, $\operatorname{rank} \Phi_{k}(S) \leq k-1$. In particular, $\Phi_{k}(S)$ is singular, that is, $\operatorname{det} \Phi_{k}(S)=0$. Now

$$
\operatorname{det} \Phi_{k}(S)=\varphi_{11} \varphi_{22} \varphi_{33} \ldots \varphi_{k k}-\varphi_{12} \varphi_{23} \ldots \varphi_{k-1, k} \varphi_{k 1},
$$

and so $\varphi_{11} \varphi_{22} \varphi_{33} \ldots \varphi_{k k}=\varphi_{12} \varphi_{23} \ldots \varphi_{k-1, k} \varphi_{k 1}$, that is, $\Gamma_{R C}(\Phi)=\Gamma_{C R}(\Phi)$. Thus we have established the following.

Lemma 11. The linear functional $\Phi$ is completely rank-nonincreasing on $\mathcal{S}_{\mathcal{P}}$ if and only if the partial matrix $\Phi$ has the cycle property (5).

We can now state and prove the main result of this section. 
Theorem 12. A linear functional defined on a pattern subspace is completely ranknonincreasing if and only if it is a limit of skew-compressions.

Proof. It is shown in [7] that a limit of skew projections is completely rank-nonincreasing. So suppose that $\Phi$ is a completely rank-nonincreasing linear functional. By Theorem $8, \Phi$ is a partial limit of rank-one matrices. Hence by Lemma 11, $\Phi$ is a limit of skew projections.

\section{REFERENCES}

[1] Johnson, C.R., Matrix completion problems: a survey. Proceedings of Symposia of Applied Mathematics, 40, Amer. Math. Soc., Providence, RI, (1990), 171-190. MR1059486

[2] Hogben, Leslie, Graph theoretic methods for matrix completion problems, Linear Alg. Appl., 328, (2001), 161-202. MR1823516 (2001k:15031)

[3] Harrison, K.J., Matrix completions and chordal graphs, International Workshop on Operator Algebras and Operator Theory, Acta Math. Sin. (Engl. Ser.), 19, (2003), 577-590. MR2014037 (2004j:15038)

[4] Rodman, Leiba, Completions of triangular matrices: a survey of results and open problems, Structured matrices in mathematics, computer science and engineering, II (Boulder, CO, 1999), Contemp. Math., 281, Amer. Math. Soc., Providence, RI, (2001), 279-293. MR1855997 (2002g:15036)

[5] Laurent, Monique, A tour d'horizon on positive semidefinite and Euclidean distance matrix completion problems, Topics in semidefinite and interior-point methods (Toronto 1996), Fields Inst. Commun. 18, Amer. Math. Soc., Providence, RI, (1998), 51-76. MR1607310 (99c:05135)

[6] Cohen, N., Johnson, C. R., Rodman, L. \& Woerdeman, H. J., Ranks of completions of partial matrices, Operator Theory: Adv. Appl., 40 (1989), 165-185. MR.1038313 (91a:15028)

[7] Hadwin, D. \& Larson, D., Completely rank nonincreasing maps, J. Funct. Anal., 199, (2003), 210-227. MR:1966828 (2004d:46066)

[8] Hou, J. \& Cui, J., Completely rank nonincreasing linear maps on nest algebras, Proc. Amer. Math. Soc., 132, (2004), 1419-1428. MR2053348(2005b:47073)

Department of Mathematics, University of New Hampshire, Durham, New Hampshire 03824

E-mail address: don@cisunix.unh.edu

School of Mathematical and Physical Sciences, Murdoch University, Murdoch, W.A. 6150, Australia

E-mail address: K.Harrison@murdoch.edu.au

Faculty of Science, Curtin University, Bentley, W.A. 6102, Australia

E-mail address: J.Ward@exchange.curtin.edu.au 